\title{
Fault-tolerant Wide-area Control for Power Oscillation Damping
}

\author{
Felix Rafael Segundo Sevilla, Student Member, IEEE, Imad Jaimoukha, Senior Member, IEEE, \\ Balarko Chaudhuri, Senior Member, IEEE, Petr Korba, Senior Member, IEEE,
}

\begin{abstract}
In this paper, the effectiveness of using both local and remote (wide-area) feedback signals for power oscillation damping (POD) controllers is shown. However, the challenge is to guarantee a minimum level of dynamic performance with only the local signal following sudden loss of remote signals. A case study on the Nordic equivalent system is presented to show that the closed-loop response could deteriorate once the remote signals are lost. A fault-tolerant control (FTC) design methodology is presented to solve this problem and ensure an acceptable performance level even in case of loss of remote signals. The FTC design methodology is based on simultaneous pole-placement for normal and loss of (remote) signals conditions along with minimisation of control effort. The problem is solved non-iteratively using Linear Matrix Inequalities (LMIs). Under the normal condition (when both local and remote signals are present) the fault-tolerant controller (FTC) requires more control effort as compared to a conventional controller (CC) in order to achieve the same performance. However, case studies on the Nordic equivalent system confirm that the proposed FTC is able to produce acceptable performance in case of loss of the remote signals while the response with a $\mathrm{CC}$ is unacceptable.
\end{abstract}

Index Terms-Power oscillation damping, fault-tolerant control, pole-placement, local and remote feedback

\section{INTRODUCTION}

$\mathbf{U}$ SE of feedback signals from geographically remote locations could improve the effectiveness of power oscillation damping (POD) [1] control. With the state-of-theart wide-area measurement systems (WAMS) infrastructure, power oscillation damping using remote (or wide-area) signals is certainly feasible. This could potentially improve the stability limits and allow operation of the transmission lines closer to their thermal capacity, without compromising security. Utilities however, are concerned about the consequences of unacceptable delay or complete loss of one or more of the remote feedback signals which could jeopardize the dynamic performance of their system.

Several techniques have been reported in the literature to tackle the adverse impact of latency or delay involved in communicating the remote signals. A list of those paper along with a critical review of the different approaches can be found in [2], [3], [4]. Another potential problem could be low data rate/bandwidth availability which is likely to be encountered

Support from ABB Switzerland under grant EESC P26939 is acknowledged.

F. R. Segundo, I. M. Jaimoukha and B. Chaudhuri are with Imperial College London, London, UK (e-mail: f.segundo-sevilla09@imperial.ac.uk, i.jaimoukha@imperial.ac.uk, b.chaudhuri@imperial.ac.uk).

P. Korba is with the ABB Corporate Research, Baden-Dättwill, Switzerland (e-mail: petrkorba@ch.abb.com). for networked communication if WAMS infrastructure were to be shared between multiple data intensive services in future. A solution to this problem was presented in [5]. Despite a number of papers on latency and less so on the bandwidth problem, very little has been reported on tackling the situation where one or more remote signals are completely lost. Impact of loss of signals on stability of inter-connected power systems is shown in [6] but no technique is presented to resolve the problem.

In this paper, the objective is to tackle the problem of loss of remote feedback signals in terms of its adverse impact on the dynamic response of the system. Following the control community's terminology, loss of remote signals is referred to as 'fault' in this paper and the proposed control technique to cater for such a 'fault' is termed 'fault-tolerant (FTC)' control. Two basic approaches to FTC are 'Fault accommodation' and 'Control reconfiguration' [7]. In 'Fault accommodation' the parameters of the controller are adapted following the loss of signal(s) while the input-output configuration remains the same (except the absence of the lost signals). On the other hand 'Control reconfiguration' an alternative set of signal(s) is employed and a revised control law is used to suit the new set. However, both these approaches requires some form of adaption rather than producing the standard linear time invariant (LTI) controllers which power utilities are well used to. Our aim here is to address the signal loss problem with a LTI fault-tolerant controller.

In this paper, a case study on the Nordic equivalent system [8] with two poorly damped inter-area modes. Supplementary power oscillation damping (POD) control of a static var compensator (SVC) [9] installed near Oslo is considered for improving the damping of the two inter-area modes. It is shown, that using only local signals, it is not possible to achieve the specified target of $10 \%$ damping ratio which justifies the use of remote signals. Using a combination of a local and a remote signal a conventional controller is shown to produce the desired performance under normal condition (i.e. when the remote signal is present). However, in case of loss of the remote signal, the system response deteriorates as in the open-loop (no POD on SVC) response which is not acceptable.

A fault-tolerant control (FTC) design methodology is proposed here to ensure an acceptable performance level even in case of loss of remote signals. The FTC design methodology is based on simultaneous pole-placement for multiple operating conditions i.e. normal (remote signals present) and loss of (remote) signals. Control effort is optimized by minimizing 
the H-infinity norm of the transfer function from external output disturbance to the control input. The resulting problem is bilinear and was solved here non-iteratively in Linear Matrix Inequalities (LMIs) framework to derive the parameters of the fault-tolerant controller.

One problem with the above technique is the combinatorial nature of the formulation when there are a number of remote signals involved. We are presently working towards addressing this issue. Another simple approach to designing a FTC would be to adopt a sequential loop closure approach where the loop is closed with the local signal first before doing the same with remote signal. Although, following loss of remote signal, this ensures at least as good a performance as with only local signal, the approach is conservative and requires more control effort than the proposed approach under normal condition. Moreover, sequential loop closure would result in higher controller orders.

The performance of the proposed fault-tolerant controller is compared against a conventional controller (CC) which is designed solely based on the normal (when both local and remote signals are present) operating condition. As expected the fault-tolerant controller (FTC) requires more control effort compared to the conventional controller (CC) in order to achieve the same performance under the normal condition. However, case studies on the Nordic equivalent system confirm that the proposed FTC is able to produce acceptable performance in case of loss of the remote signals while the response with a CC is as bad as in open loop. Thus it is a compromise between sacrificing on the control effort under normal condition in order to ensure acceptable performance under signal loss ('fault') condition.

\section{Control Design Formulation}

Consider the following state space representation of a linear time invariant (LTI) system $G(s)$ :

$$
\begin{aligned}
\dot{x}(t) & =A x(t)+B u(t) \\
y(t) & =C x(t) \\
G(s) & \stackrel{s}{=}\left[\begin{array}{l|l}
A & B \\
\hline C & 0
\end{array}\right]
\end{aligned}
$$

where $x \in \Re^{n}, u \in \Re^{q}$ and $y \in \Re^{p}$ are the state, input and output vectors, respectively. $A \in \Re^{n \times n}, B \in \Re^{n \times q}$ and $C \in$ $\Re^{p \times n}$ are the state, input and output matrices of the system, respectively. The output matrix $C$ is described as follows:

$$
C=\left[\begin{array}{lllll}
c_{1}^{T} & c_{2}^{T} & \ldots & c_{p-1}^{T} & c_{p}^{T}
\end{array}\right]^{T}
$$

where $c_{j} \in \Re^{1 \times p}$ represents the $j t h$ output of the system. In this work $p \geq 2$, as one local and at least one remote signal are used. The sensor faults or loss of signals can be represented by a family of plants $G_{i}(s)$

$$
\begin{aligned}
\dot{x}(t) & =A x(t)+B u(t) \\
y_{i}(t) & =C_{i} x(t) \\
G_{i}(s) & =\left[\begin{array}{c|c}
A & B \\
\hline C_{i} & 0
\end{array}\right]
\end{aligned}
$$

where

$$
C_{i}=\left[\begin{array}{lllll}
c_{1}^{i T} & c_{2}^{i T} & \ldots & c_{p-1}^{i T} & c_{p}^{T}
\end{array}\right]^{T}
$$

and

$$
c_{j}^{i}= \begin{cases}0 & \text { if } i=j \\ c_{j} & \text { if } i \neq j\end{cases}
$$

for

$$
\begin{aligned}
i & =0,1, \ldots, p-1 \\
j & =1,2, \ldots, p-1
\end{aligned}
$$

Note that $c_{p}^{T}$ denotes a local signal and we will assume throughout that it is always available. In nominal conditions where all signals are available, $i=0$ and $C_{0}=$ $\left[\begin{array}{lllll}c_{1}^{T} & c_{2}^{T} & \cdots & c_{p-1}^{T} & c_{p}^{T}\end{array}\right]^{T}$. Each of the measurements $y_{i}$ is the output of a sensor that can potentially fail, i.e. loss of sensor $y_{2}$ is represented as $i=2$ and is described as $C_{2}=\left[\begin{array}{lllll}c_{1}^{T} & 0 & \cdots & c_{p-1}^{T} & c_{p}^{T}\end{array}\right]^{T}$. One remote signal loss at a time is considered for simplicity but can be generalized for more than one.

\section{A. Conventional Control (CC)}

A conventional controller (CC) is designed to satisfy a desired level of dynamic performance when both the local and the remote signals are available. The performance with this controller, however, can deteriorate significantly following sudden loss of the remote signals. A regional pole-placement approach using Linear Matrix Inequalities (LMIs) is adopted for the design of CC. Unlike classical pole-placement approaches like tuning of lead-lag blocks or state feedback, the LMI approach imposition of additional constraints e.g. on control effort which is relevant to the present application.

The same methodology is used for the fault-tolerant control (FTC) design to ensure a fair comparison between FTC and CC. For the case of the CC the design formulation is linear and simpler as described below:

The CC, $K_{c}(s)$ is represented in state space form as follows

$$
\begin{aligned}
\dot{x_{c}}(t) & =A_{c} x_{c}(t)+B_{c} y(t) \\
u(t) & =C_{c} x_{c}(t) \\
K_{c}(s) & \stackrel{s}{=}\left[\begin{array}{c|c}
A_{c} & B_{c} \\
\hline C_{c} & 0
\end{array}\right]
\end{aligned}
$$

with $A_{c} \in \Re^{n \times n}, B_{c} \in \Re^{n \times p}, C_{c} \in \Re^{q \times n}$. With this controller the closed loop state dynamics is described as $\dot{\tilde{x}}=\tilde{A} \tilde{x}$ where

$$
\tilde{A}=\left[\begin{array}{c|c}
A & B C_{c} \\
\hline B_{c} C & A_{c}
\end{array}\right] .
$$

The control objective is to place the eigenvalues of (13) within a desired region of the complex plane. The following theorem taken from [10] describes the objectives:

Theorem 1: The matrix $\tilde{A}$ is stable and all its eigenvalues lie within the conic sector of the complex plane, shown in Fig. 2, if and only if there exist a symmetric matrix $\tilde{P}$ such that 


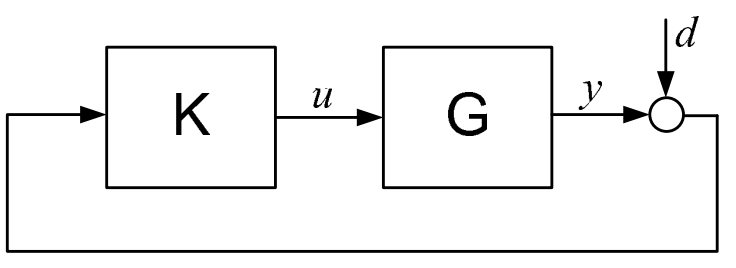

Fig. 1. Control loop with a disturbance at the plant output

$$
\tilde{P}>0
$$

$$
\left[\begin{array}{cc}
\sin \theta\left(\tilde{A^{T}} \tilde{P}+\tilde{P} \tilde{A}\right) & \cos \theta\left(\tilde{A^{T}} \tilde{P}-\tilde{P} \tilde{A}\right) \\
\cos \theta\left(\tilde{P} \tilde{A}-\tilde{A^{T}} \tilde{P}\right) & \sin \theta\left(\tilde{A^{T}} \tilde{P}+\tilde{P} \tilde{A}\right)
\end{array}\right]<0
$$

where $\theta$ is the inner angle of the cone shown in Fig. 2.

In addition to regional pole placement within the conic sector, another objective is to limit the control effort. This is achieved by minimizing the infinity norm of the transfer function between the output disturbance $d$ and the input $u$ of the system, see Figure 1. Thus the objective is to minimize $\gamma_{c}$ such that:

$$
\left\|K_{c}\left(I-G K_{c}\right)^{-1}\right\|_{\infty}<\gamma_{c}
$$

for

$$
K_{c}\left(I-G K_{c}\right)^{-1} \stackrel{s}{=}\left[\begin{array}{l|l}
\tilde{A} & \tilde{B} \\
\hline \tilde{C} & 0
\end{array}\right]
$$

where $\tilde{A} \in \Re^{2 n \times 2 n}$ is as described in (13) and $\tilde{B} \in \Re^{2 n \times p}$ and $\tilde{C} \in \Re^{q \times 2 n}$ are described below

$$
\tilde{B}=\left[\begin{array}{c}
0 \\
B_{c}
\end{array}\right] \quad \tilde{C}=\left[\begin{array}{ll}
0 & C_{c}
\end{array}\right]
$$

Applying the Bounded Real Lemma [11] to (16) we can formulate this constrains in the form of a matrix inequality

$$
\left[\begin{array}{ccc}
\tilde{A}^{T} \tilde{P}+\tilde{P} \tilde{A} & \tilde{P} \tilde{B} & \tilde{C}^{T} \\
\tilde{B}^{T} \tilde{P} & -\gamma_{c} I & 0 \\
\tilde{C} & 0 & -\gamma_{c} I
\end{array}\right]<0
$$

The formulation of this problem is bilinear but the nonlinearities can be eliminated by some appropriate change of controller variables [10]. These changes are implicitly defined in terms of the partition of the Lyapunov matrix $\tilde{P}$ and its inverse

$$
\tilde{P}=\left[\begin{array}{cc}
X & U \\
U^{T} & X_{c}
\end{array}\right] \quad \tilde{P}^{-1}=\left[\begin{array}{cc}
Y & V \\
V^{T} & Y_{c}
\end{array}\right]
$$

with $X, Y, U$ and $V \in \Re^{n \times n}$. Since $\tilde{P} \tilde{P}^{-1}=I$, consequently

$$
U V^{T}=I-X Y
$$

It has been verified that $\tilde{P}$ satisfies the identity

$$
\tilde{P} \Pi_{2}=\Pi_{1}
$$

with

$$
\Pi_{1}=\left[\begin{array}{cc}
X & I \\
U^{T} & 0
\end{array}\right], \quad \Pi_{2}=\left[\begin{array}{cc}
I & Y \\
0 & V^{T}
\end{array}\right]
$$

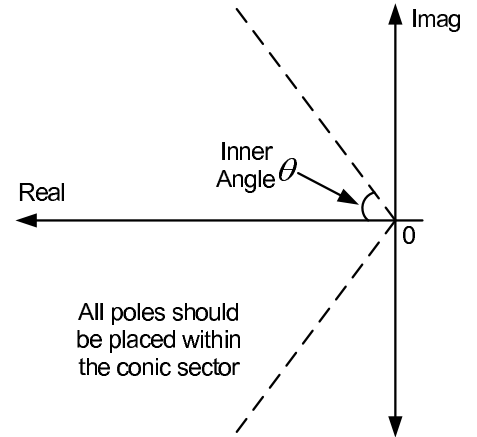

Fig. 2. Conic sector with inner angle $\theta$ where all poles should be placed

Pre- and post-multiplying (14), (15) and (19) by the matrices

$$
\begin{gathered}
\Pi_{2}^{T} \text { and } \Pi_{2}, \\
{\left[\begin{array}{cc}
\Pi_{2}^{T} & 0 \\
0 & \Pi_{2}^{T}
\end{array}\right] \text { and }\left[\begin{array}{cc}
\Pi_{2} & 0 \\
0 & \Pi_{2}
\end{array}\right],} \\
{\left[\begin{array}{ccc}
\Pi_{2}^{T} & 0 & 0 \\
0 & I & 0 \\
0 & 0 & I
\end{array}\right] \text { and }\left[\begin{array}{ccc}
\Pi_{2} & 0 & 0 \\
0 & I & 0 \\
0 & 0 & I
\end{array}\right],}
\end{gathered}
$$

respectively, carrying out the matrix products and performing the following change of variables [10]

$$
\begin{aligned}
& \hat{C}_{c}=C_{c} V^{T} \\
& \hat{B}_{c}=U B_{c} \\
& \hat{A}_{c}=X A Y+X B \hat{C}_{c}+\hat{B}_{c} C Y+U A_{c} V^{T}
\end{aligned}
$$

the constraints in (14), (15) and (19) become linear. This can be solved easily using LMI based optimization to calculate the variables of (12), which are described below:

$$
\begin{gathered}
{\left[\begin{array}{cc}
X & I \\
I & Y
\end{array}\right]>0} \\
{\left[\begin{array}{cc}
\sin \theta L_{11} & \cos \theta L_{12} \\
\cos \theta L_{12}^{T} & \sin \theta L_{11}
\end{array}\right]<0}
\end{gathered}
$$

with

$$
\left[\begin{array}{cc}
X A+A^{T} X+C^{T} \hat{B}_{c}^{T}+\hat{B}_{c} C & L_{11}= \\
* & A Y+Y A^{T}+B \hat{C}_{c}+\hat{C}_{c}^{T} B^{T}
\end{array}\right]
$$

$$
\left.\begin{array}{cc}
X A-A^{T} X+\hat{B}_{c} C-C^{T} \hat{B}_{c}^{T} & L_{12}= \\
* & \hat{A}_{c}-A^{T} \\
& A Y-Y A^{T}+B \hat{C}_{c}-\hat{C}_{c}^{T} B^{T}
\end{array}\right]
$$

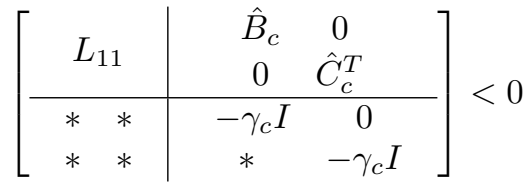

where here and elsewhere in the sequel $*$ denotes the symmetric elements in a symmetric matrix. 


\section{B. Fault-tolerant Control (FTC)}

A fault tolerant control (FTC) is designed to not only achieve a desired level of dynamic performance when both local and remote signals are available but also guarantee a minimum level of performance following sudden loss of remote signals. The problem of FTC design consists of synthesizing a single controller, if such a controller exists, that satisfies the design specifications for the family of plants as described in (7). The state space of representation of the fault tolerant controller, $K_{f}(s)$ is as follows:

$$
\begin{aligned}
\dot{x_{f}}(t) & =A_{f} x_{f}(t)+B_{f} y(t) \\
u(t) & =C_{f} x_{f}(t) \\
K_{f}(s) & \stackrel{s}{=}\left[\begin{array}{c|c}
A_{f} & B_{f} \\
\hline C_{f} & 0
\end{array}\right]
\end{aligned}
$$

with $A_{f} \in \Re^{n \times n}, B_{f} \in \Re^{n \times p}$ and $C_{f} \in \Re^{q \times n}$. This controller is designed for the family of plants (7) such that:

$$
\begin{aligned}
u(s)=K_{f}(s) y_{0}(s), u(s) & =K_{f}(s) y_{1}(s) \\
\cdots, u(s) & =K_{f}(s) y_{p}(s)
\end{aligned}
$$

and the closed loop state dynamics matrices are given by:

$$
\tilde{A}_{i}=\left[\begin{array}{c|c}
A & B C_{f} \\
\hline B_{f} C_{i} & A_{f}
\end{array}\right], i=0,1, \ldots, p .
$$

The requirement is that the eigenvalues of all $\tilde{A}_{i}$ lie in the conic region described in Fig. 2. The constraints are the same as in CC and Theorem 1 is used to formulate the problem. The objective in this case is to find a symmetric $\tilde{P}$ such that:

$$
\begin{gathered}
\tilde{P}>0 \\
{\left[\begin{array}{cc}
\sin \theta_{i}\left(\tilde{A_{i}^{T}} \tilde{P}+\tilde{P} \tilde{A}_{i}\right) & \cos \theta_{i}\left(\tilde{A_{i}^{T}} \tilde{P}-\tilde{P} \tilde{A}_{i}\right) \\
\cos \theta_{i}\left(\tilde{P} \tilde{A}_{i}-\tilde{A}_{i}^{T} \tilde{P}\right) & \sin \theta_{i}\left(\tilde{A}_{i}^{T} \tilde{P}+\tilde{P} \tilde{A}_{i}\right)
\end{array}\right]<0,} \\
i=0,1, \ldots, p
\end{gathered}
$$

In this case, $\theta_{i}$ is the required inner angle for the $i$ th system. Similarly to CC, another objective is to limit the control effort which is included in the formulation below:

$$
\left\|K_{f}\left(I-G_{i} K_{f}\right)^{-1}\right\|_{\infty}<\gamma_{i f}, i=0,1, \ldots, p .
$$

We interpret $\gamma_{i f}, i=1,2, \ldots, p$, as apriori constraints on the control effort for the 'fault' scenarios (when remote signals are not available) and the objective is to minimize $\gamma_{0 f}$, which corresponds to the control effort for the normal (both local and remote signals available) scenario. The Bounded Real Lemma is used again to express (42) for each $i$ in a matrix inequality form

$$
\left[\begin{array}{ccc}
\tilde{A}_{i}^{T} \tilde{P}+\tilde{P} \tilde{A}_{i} & \tilde{P} \tilde{B} & \tilde{C}^{T} \\
\tilde{B}^{T} \tilde{P} & -\gamma_{i f} I & 0 \\
\tilde{C} & 0 & -\gamma_{i f} I
\end{array}\right]<0
$$

Applying the same transformations to (40), (41) and (43) as before and carrying out the corresponding matrix products, it is not possible to perform the same change of variables as in (27), (28) and (29) to linearize the inequalities due to the problem of having multiple systems. In order to linearize the bilinearities, after applying the transformations (24), (25) and (26) to (40), (41) and (43) respectively, we apply the following change of variables [12]

$$
\begin{gathered}
L=C_{f} V^{T} \quad F=U B_{f} \quad M^{T}=U A_{f} V^{T} \\
P=Y^{-1} \quad S^{T}=M^{T} Y^{-1}
\end{gathered}
$$

which have dimensions determined by the transformations. Defining $\hat{A}=A+B C_{f}$, the final formulation of the problem is represented by the following inequalities

$$
\begin{gathered}
{\left[\begin{array}{cc}
P & P \\
P & X
\end{array}\right]>0} \\
{\left[\begin{array}{cl}
\sin \theta_{i} L_{11}^{i} & \cos \theta_{i} L_{12}^{i} \\
* & \sin \theta_{i} L_{11}^{i}
\end{array}\right]<0}
\end{gathered}
$$

where

$$
\begin{aligned}
L_{11}^{i} & =\left[\begin{array}{cc}
\hat{A}^{T} P+P \hat{A} & P A+\hat{A}^{T} X+C_{i}^{T} F^{T}+S \\
* & A^{T} X+X A+C_{i}^{T} F^{T}+F C_{i}
\end{array}\right] \\
L_{12}^{i} & =\left[\begin{array}{cc}
\hat{A}^{T} P-P \hat{A} & -P A+\hat{A}^{T} X+C_{i}^{T} F^{T}+S \\
* & A^{T} X-X A+C_{i}^{T} F^{T}-F C_{i}
\end{array}\right]
\end{aligned}
$$

and

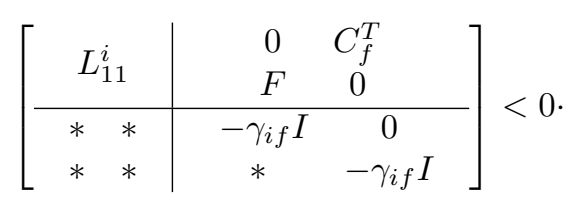

For further details see [12]. The algorithm to calculate the parameters of the FTC in (37) is outlined below:

- Define the desired damping required $\zeta_{i}$ and calculate $\theta_{i}=\cos ^{-1}\left(\zeta_{i}\right)$.

- Define the desired control effort levels for $\gamma_{1 f}, \ldots, \gamma_{p f}$

- Build and solve $Y>0$

$$
\left[\begin{array}{cc}
\sin \theta_{i}\left(A^{T} Y+Y A\right. & \cos \theta_{i}\left(Y A-A^{T} Y\right. \\
\left.+B L+L^{T} B^{T}\right) & \left.+L^{T} B^{T}-B L\right) \\
* & \sin \theta_{i}\left(A^{T} Y+Y A\right. \\
* & \left.+B L+L^{T} B^{T}\right)
\end{array}\right]<0
$$

- Get $Y$ and $L$ from (51) and calculate $C_{f}=L Y^{-1}$.

- Define $\hat{A}=A+B C_{f}$.

- Minimize $\gamma_{0 f}$ subject to (46), (47) and (50) to get $P, X$, $F$ and $S$.

- Using $V=V^{T}=Y$, (21), (44) and (45) calculate $B_{f}$ and $A_{f}$.

- Define $K_{f} \stackrel{s}{=}\left(A_{f}, B_{f}, C_{f}, 0\right)$.

\section{TEST SySTEM}

A reduced equivalent of the Nordic system is used for the case study. The detailed model with approximately 3000 buses, 4000 branches and 1100 generators [13], [8] was reduced down to a 20 generators, 36 bus equivalent system shown in Fig. 3. The aim was to retain the modal behavior of the two most critical poorly damped inter-area modes. A static VAr compensator (SVC) is included in the model at Hasle substation (5101) in south-east Norway in the same way it exists in practice. The total number of state variables for the linearized version of the reduced equivalent system is 296 . 


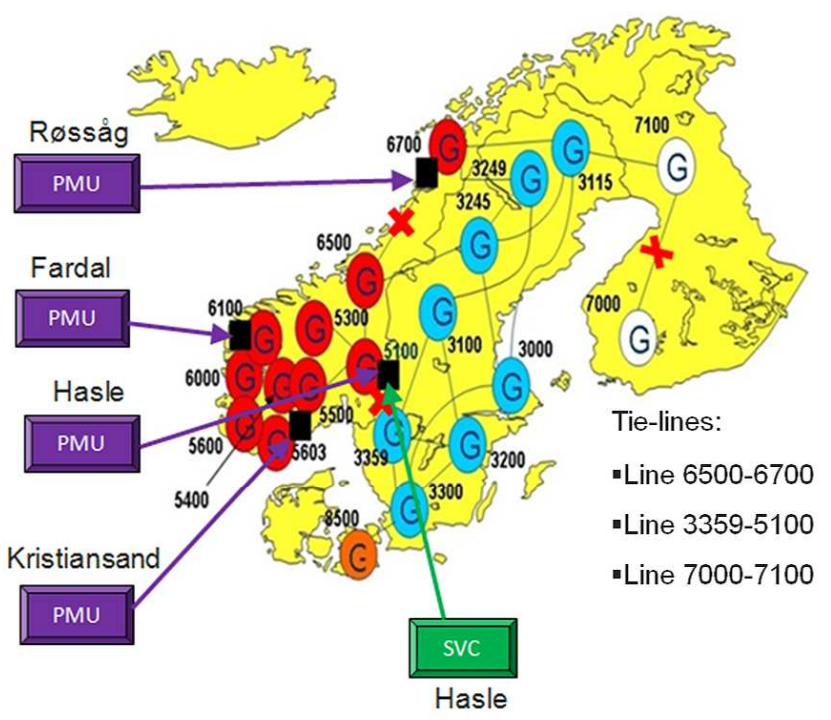

Fig. 3. Nordic equivalent system where the location of the PMUs and key tie-lines are marked.

There are two critical inter-area modes, 0.29 and $0.55 \mathrm{~Hz}$ with $4.8 \%$ and $5.4 \%$ damping, respectively, under nominal condition, see Table I. The first mode $(0.29 \mathrm{~Hz})$ comprises of the Finnish generators swinging against the rest whereas the second mode $(0.55 \mathrm{~Hz})$ involves the generators in the north of Finland, Sweden and Norway swinging against those in the southern parts of these countries.

Following critical contingencies like outage of one of the parallel lines connecting (i) 6500-6700 (Norwegian coast line), (ii) 7100-7000 (Finnish line) or (iii) 3359-5101 (Hasle line), the damping of the inter-area modes could be as low as $2-3 \%$ requiring improvement through supplementary control. Here the challenge is to ensure satisfactory damping of both the modes by designing a POD for the SVC at Hasle.

With a number of phasor measurement units (PMUs) (only four in Norway and two in Finland are considered for this study) installed throughout the Nordic system remote feedback signals were also considered in addition to the local signals. The difference between voltage angles available from the PMUs were chosen as potential candidates [13] for remote signals. The magnitude and phase angle of the residues for each candidate is shown in Table II. Voltage angle difference between the PMUs at 6100 (at Nedre Rossaga) and 7000 (in southern Finland) has high residue magnitude for both modes and the phase angles are also in the same direction unlike the case of 6700-7000 where those are in opposite directions. Thus based on both magnitude and phase angle of the residues [14], 6100-7000 (shown in boldface in Table II) was selected as the most appropriate signal for the POD.

Out of the available local signals, magnitude and phase angle of the line currents were found to be the most effective candidates. The magnitude and phase angle of the residues associated with the local current signals are shown in Table III. Phase angle of current in line 5101-5501 (shown in boldface in Table III) was selected as the most appropriate local signal based on both the magnitude and phase angle of the residue.
TABLE I

DAMPING AND FREQUENCIES OF THE INTER-AREA MODES UNDER DIFFERENT SCENARIOS

\begin{tabular}{|c|c|c|}
\hline \multicolumn{3}{|c|}{ Open Loop Nominal Conditions } \\
\hline Mode No. & $\begin{array}{c}\text { Frequency } \\
(\mathrm{Hz})\end{array}$ & $\begin{array}{c}\text { Damping Ratio } \\
(\zeta)\end{array}$ \\
\hline Mode 1 & 0.29 & 0.049 \\
Mode 2 & 0.55 & 0.054 \\
\hline
\end{tabular}

TABLE II

REMOTE SIGNAL SELECTION: MAGNITUDE AND PHASE ANGLE OF RESIDUES FOR DIFFERENCE BETWEEN RELEVANT VOLTAGE ANGLES

\begin{tabular}{|c|c|c|c|c|}
\hline & \multicolumn{2}{|c|}{ Mode 1} & \multicolumn{2}{|c|}{ Mode 2} \\
\hline Signal & Mag & Angle (deg) & Mag & Angle (deg) \\
\hline $5101-5603$ & 0.03 & -64 & 0.68 & -80 \\
\hline $5101-7000$ & 2.28 & 110 & 1.55 & 83 \\
\hline $5101-7100$ & 1.26 & 107 & 1.97 & 94 \\
\hline $5603-6700$ & 0.34 & 109 & 3.41 & 97 \\
\hline $5603-7100$ & 1.29 & 107 & 2.65 & 96 \\
\hline $6100-7000$ & 2.32 & 110 & 2.44 & 88 \\
\hline $6700-7000$ & 1.97 & 111 & 1.26 & -67 \\
\hline $7000-7100$ & 1.02 & -66 & 0.53 & 127 \\
\hline
\end{tabular}

TABLE III

Local SignaLs: MAgNitUde AND PHASE ANGLE OF RESIDUES FOR CURRENTS

\begin{tabular}{|c|c|c|c|c|}
\hline & \multicolumn{2}{|c|}{ Mode 1} & \multicolumn{2}{|c|}{ Mode 2} \\
\hline Signal & Mag & Angle (deg) & Mag & Angle (deg) \\
\hline \begin{tabular}{|l|l|}
$I$ & $3359-5101$
\end{tabular} & 0.28 & -62.09 & 4.84 & -81.44 \\
\hline$I_{\theta} 3359-5101$ & 0.35 & 100.43 & 0.35 & 44.44 \\
\hline$|I| 5101-5501$ & 0.21 & -64.84 & 3.76 & -82.20 \\
\hline$I_{\theta} \mathbf{5 1 0 1}-\mathbf{5 5 0 1}$ & 0.73 & 113 & 6.31 & $\mathbf{9 7 . 5 2}$ \\
\hline$|I| 5100-5100$ & 0.38 & -56.46 & 5.52 & -80.70 \\
\hline$I_{\theta} 5100-5100$ & 0.48 & 104.51 & 2.27 & 89.24 \\
\hline
\end{tabular}

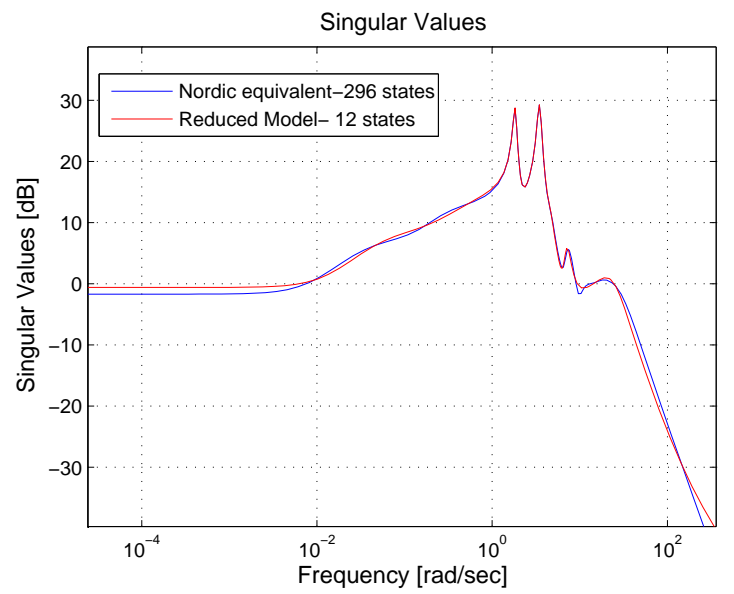

Fig. 4. Frequency response of the original and reduced model

\section{Control Design}

The control design formulation presented in Sections II-A and II-B produces controllers that are of the same size (dimension) as the plant. To get around this problem, linearized model of the test system (described in Section III) with 296 states was first reduced to a 12th order equivalent using balanced stochastic model truncation through Schur method [15]. The similarity between the frequency responses of the original and reduced system, shown in Figure 4, confirms the validity of this approach.

Two case studies with three different controllers (CC using 
local signal, CC using local and remote signals and FTC), each of order 12, are presented in this paper. For the first case, the local signal $I_{\theta} 5101-5501$ was used to design a CC following the formulation in Section II-A. Although the design specification was to achieve a minimum $10 \%$ damping for both inter-area modes, using the local signal only $8 \%$ damping could be achieved. Using both local and remote signals, the target $10 \%$ damping could be achieved for both the modes.

In the next case study, the performance of the $\mathrm{CC}$ with local and remote feedback was compared against a FTC designed using the approach described in Section II-B. Like CC, the FTC also improves the damping of the two critical modes up to $10 \%$ under normal conditions. In addition, the FTC maintains the damping above $8 \%$ without the remote signal. However, with CC the damping of mode 1 reduces to to $4.9 \%$ in absence of the remote signal. This can be explained from Table III which shown that the residue for the chosen local signal is reasonable for mode 2 but poor for mode 1 .

Both CC and the FTC were designed to minimize the control effort in terms of the infinity norms of (16) and (42). The minimum values of $\gamma$ (see (16), (42)) was less for CC than FTC which is also reflected in the difference in control effort in Fig. 6(c).

\section{Case Study I: Local and Remote Feedback SIGNALS}

In this section the effectiveness of using both local and remote (wide-area) feedback signals for power oscillation damping (POD) control is demonstrated. This is compared against the case where only local signal is used. The two scenarios are compared in terms of the dynamic responses shown in Fig. 5 following a short circuit and outage of the tie-line 6500-6700 at 5 secs.

Subplots Fig. 5(a) and Fig. 5(b) show the power flow through the tie lines 3359-7100 and 7000-7100 where the oscillations due to the two critical inter-area modes (described in Table I) are clearly visible. Oscillation due to mode 2 is prominent in the power flow through the line 3359-5101 while the effect of mode 1 is primarily visible in the power flow through line 7000-7100. It can be seen that using both local and remote signals (Loc+Rem), the oscillations settle quicker than using only local signal (Loc).

The susceptance $B_{S V C}$ (Fig. 5(c)) of the SVC and the bus voltage (Fig. 5(d)) at the Bus 5101 where the SVC is installed are also shown. Combination of local and remote signals requires higher variations $B_{S V C}$ (control effort) and consequently introduces higher variations in the voltage (Fig. 5(d)). However, the variations in bus voltage and susceptance of the SVC are both within the specified limits.

\section{Vi. Case Study II: Fault-tolerant Controller}

In this section the dynamic performance with the faulttolerant controller (FTC) is compared against a conventional controller (CC) under both normal (when remote signal is present) and 'fault' (when remote signal is lost) conditions. Both the FTC and CC were designed using the same local and remote feedback signals mentioned in Section III. The
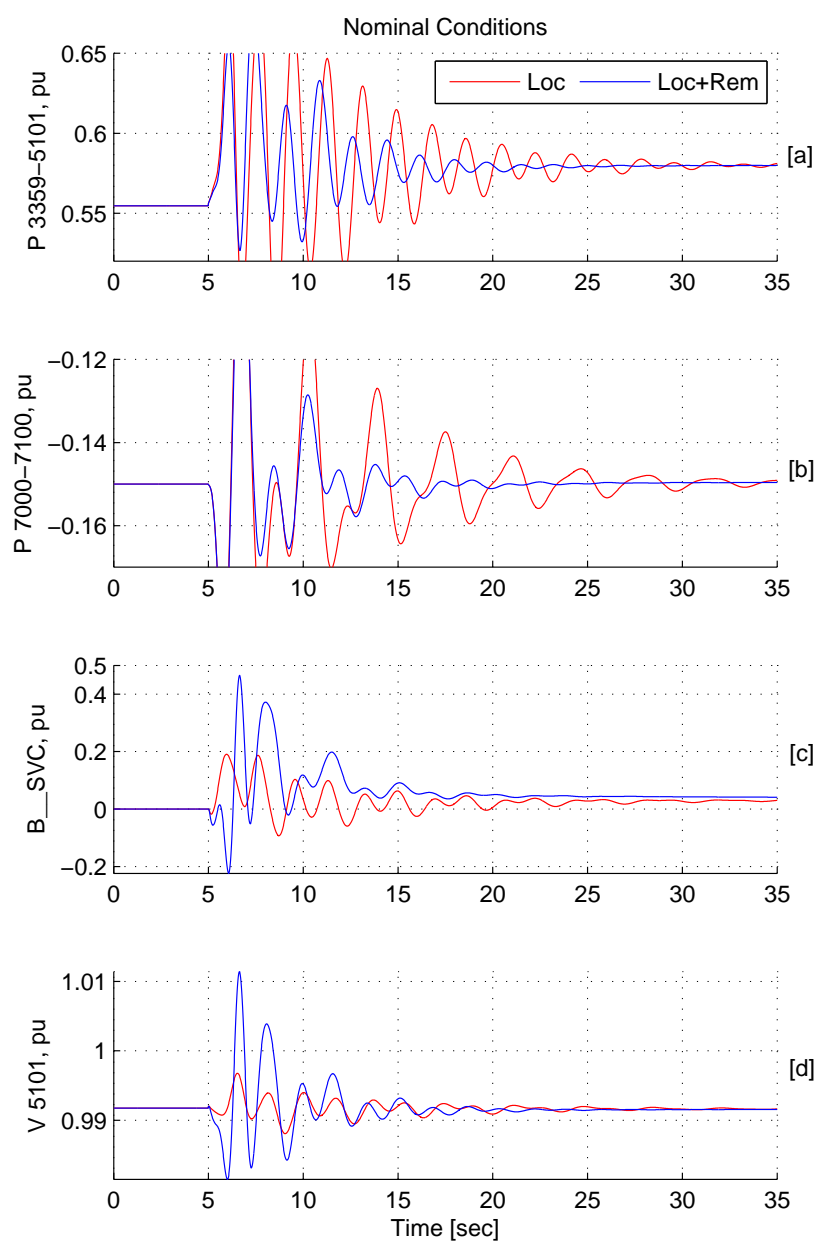

Fig. 5. Comparison of dynamic performance with local (Loc) and a combination of local and remote (Loc + Rem) signals

design specification in both cases was to achieve a minimum $10 \%$ damping while minimizing the control effort as outlined in Sections II-A and II-B. However, for the FTC, the target damping was reduced to $8 \%$ for the 'fault' condition to obtain a feasible solution. A short circuit followed by outage of tieline 6500-6700 (same as in Case Study I) was created to compare the dynamic performance with $\mathrm{CC}$ and FTC.

\section{A. Normal Condition}

Under normal condition, the dynamic performance with FTC and CC as seen from the power flows through the lines 3359-5101 (Fig. 6(a)) and 7000-7100 (Fig. 6(b)) are similar. However, higher control effort is required by the FTC as indicated by larger excursion in $B_{S V C}$ (Fig. 6(c)) and also the bus voltage (Fig. 6(d)). This is in agreement with relative values of $\gamma_{c}$ and $\gamma_{0 f}$ for the two cases which is an implicit measure of control effort as described in Sections II-A and II-B.

Thus under normal condition, $\mathrm{CC}$ is preferable from control effort point of view even though both CC and FTC produce similar dynamic performance. 

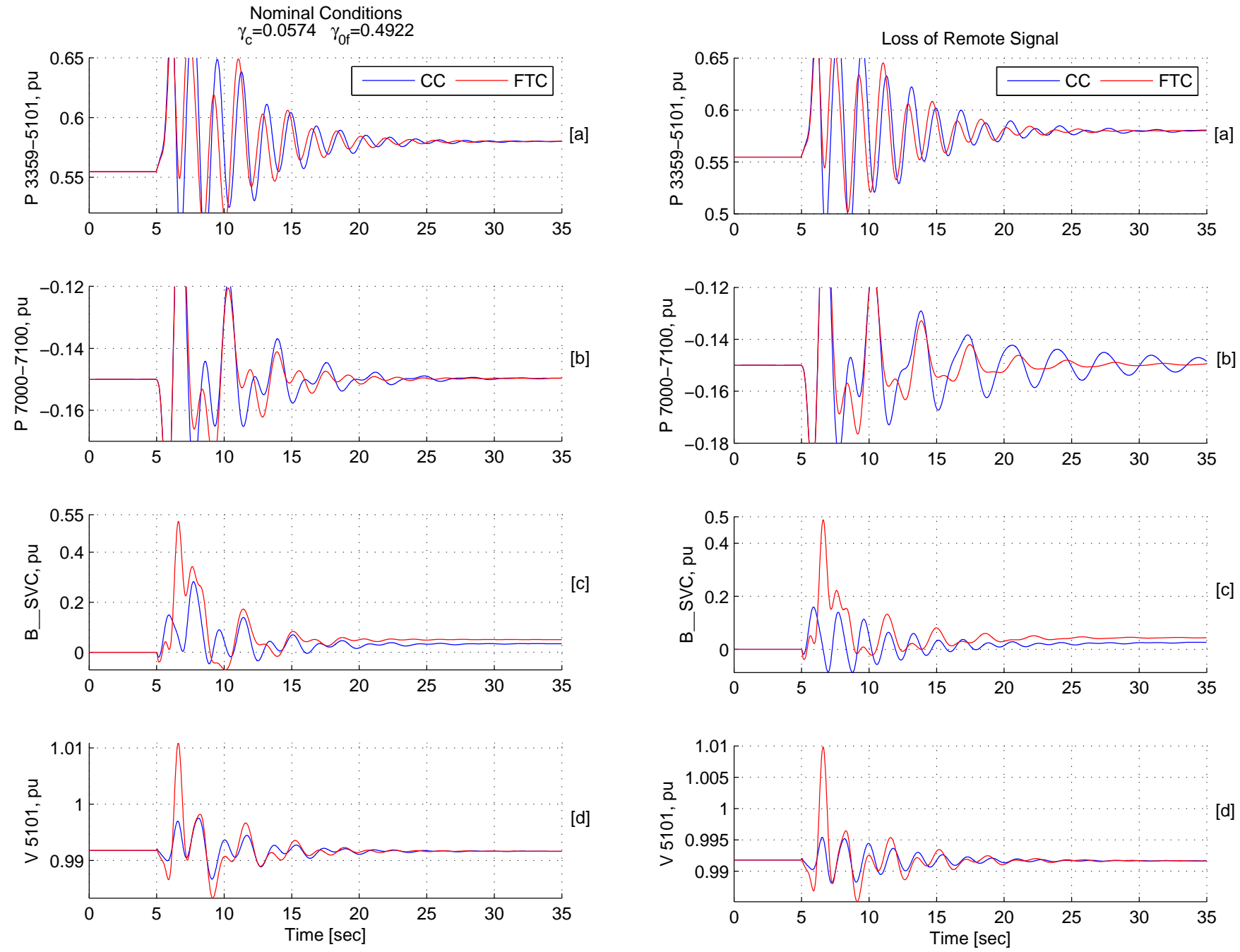

Fig. 6. Comparison of dynamic performance with CC and FTC under normal (remote signal present) condition

Fig. 7. Comparison of dynamic performance with CC and FTC following loss of remote signal

\section{B. Loss of Remote Signals}

to significant deterioration in dynamic behavior.

The performance with $\mathrm{CC}$ is compared against FTC in Fig. 7 in the face of loss of remote signal.

The variation of the power flow through line 3359-5101 (Fig. 7(a)) is not affected much by the loss of the remote signal for both CC and FTC. As mentioned before, this power flow predominantly reflects mode 2 which has adequate observability (residue) in the local signal which explains the above trend. On the other hand, power flow through 7000-7100 has prominent presence of mode 1 which is not adequately observable in the local signal. Hence, with CC, loss of the remote signal results in significant deterioration in the oscillatory behavior of this signal (Fig. 7(b)). However, with FTC, satisfactory performance is maintained for both the powerflows with only the local signal. Similar to the normal condition, the control effort required by the FTC is higher than $\mathrm{CC}$ as evident through larger excursions on $B_{S V C}$ (Fig. 7(c)).

To summarize, although the FTC requires larger control effort than $\mathrm{CC}$ to achieve a desired damping under normal condition, the former can guarantee an acceptable performance level when the remote signal is lost unlike the $\mathrm{CC}$ which leads

\section{CONCLUSION}

The effectiveness of using both local and remote (widearea) feedback signals for power oscillation damping (POD) controllers has been demonstrated. However, the challenge is to guarantee an acceptable dynamic performance with only the local signal following sudden loss of remote signals. A case study on the Nordic equivalent system shows that the closed-loop response could deteriorate once the remote signals are lost. A fault-tolerant control (FTC) design methodology is presented to solve this problem and ensure an acceptable performance level even in case of loss of remote signals. The FTC design methodology is based on simultaneous poleplacement for normal and loss of (remote) signals conditions along with minimisation of control effort. Under the normal condition (when both local and remote signals are present) the fault-tolerant controller (FTC) requires more control effort as compared to a conventional controller (CC) in order to achieve the same performance. However, case studies on the Nordic equivalent system confirm that the proposed FTC is able to 
produce acceptable performance in case of loss of the remote signals while the response with a CC is unacceptable.

Although we have presented simulations based on the nonlinear model to illustrate our approach, we should emphasize that complete stability region information can only be obtained by taking into account the nonlinearity of the underlying nonlinear system and this is a topic of future research. Also, a couple of challenges with the proposed faulttolerant controller is conservativeness and complexity due to combinatorial nature of the problem when multiple remote signals are involved. However, the major advantage is that the resulting FTC is obtained in simple linear time-invariant (LTI) form which power utilities are well used to rather than an adaptive structure.

\section{ACKNOWLEDGEMENT}

Funding from ABB, Switzerland (Grant EESC P26939) and technical input from people within $\mathrm{ABB}$ are gratefully acknowledged.

\section{REFERENCES}

[1] I. Kamwa, A. Heniche, G. Trudel, M. Dobrescu, R. Grondin, and D. Lefebvre, "Assessing the technical value of FACTS-based wide-area damping control loops," in proceedings of IEEE Power Engineering Society General Meeting, 2005, 2005, pp. 1734-1743 Vol. 2.

[2] D. Dotta, A. S. e Silva, and I. C. Decker, "Wide-area measurementsbased two-level control design considering signal transmission delay," IEEE Transactions on Power Systems, vol. 24, no. 1, pp. 208-216, 2009.

[3] B. Chaudhuri, R. Majumder, and B. Pal, "Wide-area measurement-based stabilizing control of power system considering signal transmission delay," IEEE Transactions on Power Systems, vol. 19, no. 4, pp. 19711979, 2004.

[4] N. R. Chaudhuri, S. Ray, R. Majumder, and B. Chaudhuri, "A new approach to continuous latency compensation with adaptive phasor power oscillation damping controller (pod)," IEEE Transactions on Power Systems, vol. 25, no. 2, pp. 939-946, 2010.

[5] N. R. Chaudhuri, D. Chakraborty, and B. Chaudhuri, "An architecture for FACTS controllers to deal with bandwidth-constrained communication," IEEE Transactions on Power Delivery, vol. 26, no. 1, pp. 188-196, 2011.

[6] K. Mekki, A. Snyder, N. HadjSaid, R. Feuillet, D. Georges, and T. Margotin, "Damping controller input-signal loss effects on the widearea stability of an interconnected power system," in IEEE Power Engineering Society Summer Meeting, vol. 2, 2000, pp. 1015-1019.

[7] M. Blanke, M. Kinnaert, J. Lunze, M. Staroswiecki, and J. Schroeder, Diagnosis and fault-tolerant control, 2nd ed. Berlin; New York: Springer, 2006.

[8] N. Chaudhuri, A. Domahidi, R. Majumder, B. Chaudhuri, P. Korba, S. Ray, and K. Uhlen, "Wide-area power oscillation damping control in nordic equivalent system," Generation, Transmission Distribution, IET, vol. 4, no. 10, pp. $1139-1150$, october 2010.

[9] N. G. Hingorani and L. Gyugyi, Understanding FACTS: concepts and technology of flexible AC transmission systems. New York: IEEE Press, 2000.

[10] M. Chilali and P. Gahinet, "H-infinity design with pole placement constraints: an lmi approach," Automatic Control, IEEE Transactions on, vol. 41, no. 3, pp. $358-367$, mar 1996.

[11] M. Chilali, P. Gahinet, and P. Apkarian, "Robust pole placement in Imi regions," Automatic Control, IEEE Transactions on, vol. 44, no. 12, pp. $2257-2270$, dec 1999.

[12] R. Ramos, L. Alberto, and N. Bretas, "A new methodology for the coordinated design of robust decentralized power system damping controllers," Power Systems, IEEE Transactions on, vol. 19, no. 1, pp. $444-454$, feb. 2004.

[13] E. Johansson, K. Uhlen, A. Leirbukt, P. Korba, J. Gjerde, and L. Vormedal, "Coordinating power oscillation damping control using wide area measurements," in Power Systems Conference and Exposition, 2009. PSCE '09. IEEE/PES, march 2009, pp. 1 -8.
[14] S. Ray, B. Chaudhuri, and R. Majumder, "Appropriate signal selection for damping multi-modal oscillations using low order controllers," in proceedings of IEEE Power Engineering Society General Meeting, 2008, Pittsburgh, 2008.

[15] M. G. Safonov and R. Y. Chiang, "Model reduction for robust control: A schur relative-error method," in American Control Conference, 1988, june 1988 , pp. $1685-1690$.

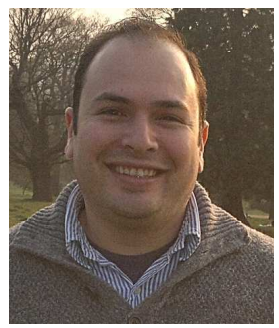

Felix Rafael Segundo Sevilla was born in Zamora, Mexico. He received his BSc in Electrical Engineering from the Instituto Tecnologico de Morelia, and an MSc from the Universidad Autonoma de Nuevo Leon, both in Mexico, in 2005 and 2007 respectively. In 2008, he joined ABB corporate research in Switzerland for one year term internship where he worked under the supervision of Dr. Petr Korba modelling and analysing power systems. Currently he is pursuing a $\mathrm{PhD}$ at Imperial College London, $\mathrm{UK}$ in a project funded by $\mathrm{ABB}$. His research interests include power systems dynamics and stability, robust and fault tolerant control.

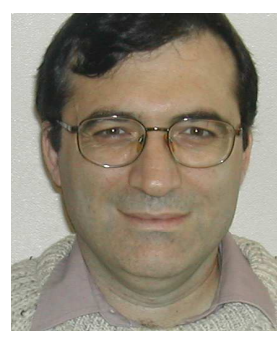

Imad Jaimoukha Imad M. Jaimoukha was born in Amman, Jordan and received his BSc in Electrical Engineering from the University of Southampton, England in 1983. He received an $\mathrm{MSc}$ and a $\mathrm{PhD}$ in Control Systems from Imperial College, London in 1986 and 1990, respectively. He worked as a Research Fellow at the Centre for Process Systems Engineering at Imperial College from 1990 till 1994. Since 1994 he has been with the Department of Electrical and Electronic Engineering, Imperial. His research interests include robust control, system approximation, fault detection and isolation, control for power systems and global optimization.

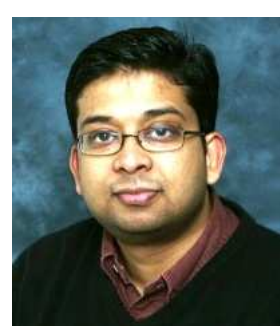

Balarko Chaudhuri (S'02-M'06-SM'11) received his $\mathrm{PhD}$ from Imperial College London in 2005. He worked with General Electric Global Research for a year before coming back to Imperial College as a post-doctoral research associate in 2006 . He is presently a lecturer in the department of Electrical and Electronic Engineering at Imperial College London. His research interests are in the areas of power system dynamics and stability, FACTS/HVDC, robust control and renewable energy.

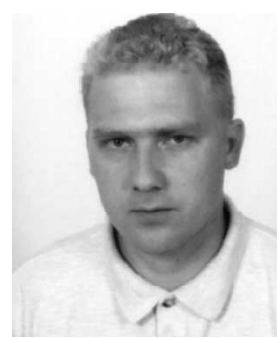

Petr Korba received his MSc in electrical engineering from the Czech Technical University, Prague, Czech Republic, in 1995 and his $\mathrm{PhD}$ from the University of Duisburg, Germany, in 2000. He was an invited scientist at the Delft University of Technology, the Netherlands, and at the University of Manchester Institute of Science and Technology (UMIST) in 1998 and 1999, respectively. He became a member of staff at UMIST, Control Systems Centre, where he stayed until 2001. Since then he has been with ABB Switzerland Ltd. His interests include model identification techniques, robust and adaptive control theory and their industrial applications. 